\section{Mehr Allergien bei Frühchen?}

\section{Frühgeborene oder Kinder mit niedrigem Geburtsgewicht nehmen über den Darm bis zu 100-mal mehr potenziell Allergien auslösende Proteine auf als Kinder, die zum Termin bzw. mit Normalgewicht geboren werden. Resultiert daraus eine höhere Inzidenz an Nahrungsmittelallergien?}

n der Manitoba Birth Cohort Data-

base sind insgesamt 16.320 Neugeborene registriert, die im Jahr $1995 \mathrm{im}$ kanadischen Bundesstaat Manitoba geboren wurden. 13.980 von ihnen konnten bis zum Jahr 2002 weiter beobachtet werden. 881 dieser Kinder (6,3\%) waren vor Ende der 37. Schwangerschaftswoche zur Welt gekommen. Insgesamt 691 (4,9\%) der Neugeborenen hatten bei Geburt ein Gewicht unter $2,5 \mathrm{~kg}$.

Im Verlauf von sieben Jahren wurde bei 592 Kindern $(4,2 \%)$ die Diagnose „Nahrungsmittelallergie“ gestellt, 316 Kinder $(2,3 \%)$ hatten von ihren Ärzten aufgrund der Gefahr einer anaphylaktischen Reaktion injizierbares Adrenalin verordnet bekommen. Patienten mit Hymenopterengiftallergie wurden nicht mitgerechnet.

Beim Vergleich der termingerecht geborenen Kinder mit den Frühgebore- nen ergab sich kein signifikanter Hinweis auf ein unterschiedliches Risiko für eine Nahrungsmittelallergie. Das gleiche galt für den Vergleich von Neu-

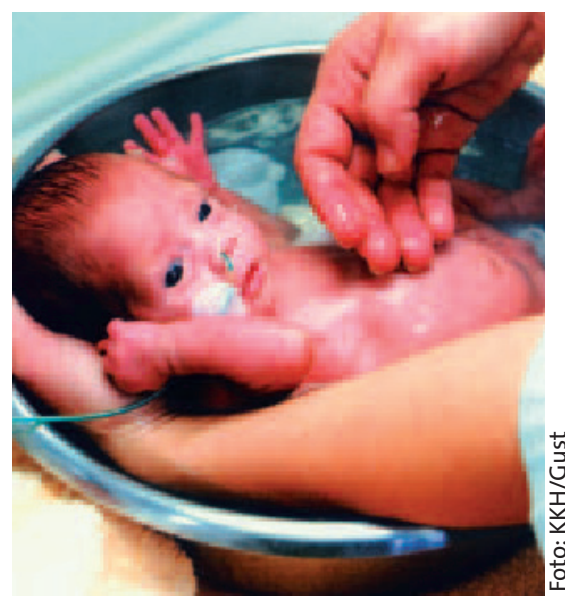

Ein zu früher Start ins Leben bedeutet nicht automatisch auch mehr Allergien im späteren Leben. geborenen mit einem Geburtsgewicht von unter $2,5 \mathrm{~kg}$ mit Kindern mit Normalgewicht bei der Geburt. Auch in den einzelnen Untergruppen, aufgeschlüsselt nach genauem Gestationsalter oder Geburtsgewicht, war in keinem Fall ein erhöhtes Allergierisiko nachzuweisen. Als statistisch signifikante Risikofaktoren für die Entstehung einer Nahrungsmittelallergie konnten vielmehr folgende Faktoren identifiziert werden: Asthma oder Nahrungsmittelallergie der Mutter, männliches Geschlecht, Wohnen in städtischer Umgebung und überdurchschnittliches Einkommen der Eltern.

Fazit: Die Daten dieser großen Kohortenstudie zeigen, dass Frühgeborene oder Neugeborene mit zu niedrigem Gewicht später kein höheres Risiko für eine Nahrungsmittelallergie aufweisen als termingerecht bzw. normalgewichtig geborene Säuglinge. Die bei diesen Neugeborenen nachgewiesene höhere Darmpermeabilität scheint Nahrungsmittelallergien nicht zu begünstigen.

af

Liem JJ et al. The risk of developing food allergy in premature or low-birth-weight children. J Allergy Clin Immunol 2007; 119: 1203-9

tät gewonnene Seren von 21 Kindern mit einer persistierenden Hühnereiweißallergie und von 16 Kindern mit einer Toleranzentwicklung in den ersten Lebensjahren auf Antikörper gegen Hühnereiproteine und unterschiedliche Ovomucoid-Epitop-Bindungsmuster. Eingesetzt wurden Immunoblots oder Immuno-CAPs mit nativen Allergenen mit erhaltener Konformation sowie mit chemisch reduzierten und alkylierten und dadurch „linearisierten“ (sequenziellen) Hühnereiallergenen.

Es zeigte sich, dass Kinder mit einer persistierenden Hühnereiallergie höhere IgE-Antikörperkonzentrationen gegen sequenzielles und natives Ovomucoid und Ovalbumin im Vergleich zu den Kindern mit einer wieder verschwundenen Allergie hatten $(\mathrm{p}<0,01)$. Im Ovomucoidallergen wurden vier Major-IgEBindungsstellen identifiziert, welche 